\title{
Localized States in Bi-Pattern Systems
}

\author{
U. Bortolozzo, ${ }^{1}$ M. G. Clerc, ${ }^{2}$ F. Haudin, ${ }^{1}$ R. G. Rojas, ${ }^{3}$ and S. Residori ${ }^{1}$ \\ ${ }^{1}$ l'Institut Non Linéaire de Nice (INLN), Centre National de la Recherche Scientifique (CNRS), \\ Université de Nice Sophia Antipolis, 1361 Route des Lucioles, 06560 Valbonne, France \\ ${ }^{2}$ Departamento de Física, Facultad de Ciencias Físicas y Matemáticas, Universidad de Chile, Casilla 487-3, \\ Blanco Encalada 2008, Santiago, Chile \\ ${ }^{3}$ Instituto de Física, Pontificia Univesidad Católica de Valparaíso, Casilla 4059, Avenida Brasil 2950, Valparaíso, Chile
}

Correspondence should be addressed to M. G. Clerc, marcel@dfi.uchile.cl

Received 4 February 2009; Accepted 7 May 2009

Recommended by M. Tlidi

We present a unifying description of localized states observed in systems with coexistence of two spatially periodic states, called $b i$-pattern systems. Localized states are pinned over an underlying lattice that is either a self-organized pattern spontaneously generated by the system itself, or a periodic grid created by a spatial forcing. We show that localized states are generic and require only the coexistence of two spatially periodic states. Experimentally, these states have been observed in a nonlinear optical system. At the onset of the spatial bifurcation, a forced one-dimensional amplitude equation is derived for the critical modes, which accounts for the appearance of localized states. By numerical simulations, we show that localized structures persist on twodimensional systems and exhibit different shapes depending on the symmetry of the supporting patterns.

Copyright (c) 2009 U. Bortolozzo et al. This is an open access article distributed under the Creative Commons Attribution License, which permits unrestricted use, distribution, and reproduction in any medium, provided the original work is properly cited.

\section{Introduction}

Spatial patterns appear spontaneously in out-of-equilibrium systems and are observed in many different physical contexts [1]. During the last two decades, spatial pattern formation has been largely studied, leading to the identification of various types of spatiotemporal instabilities and symmetry selection processes in the general frameworks of dynamical systems and bifurcation theory [2, 3]. Localized structures, that is, patterns extended over a restricted spatial domain, have received, in particular, a large interest, and from the early observations of magnetic domains in ferromagnetic materials [4], localized states have been successively observed in such different systems as liquid crystals [5], plasmas [6], chemical reactions [7], fluid surface waves [8], granular media $[9,10]$, and thermal convection $[11,12]$. In nonlinear optics localized structures were first predicted as solitary waves in bistable optical cavities [13], and successively also explained in terms of diffractive auto-solitons [14]. Optical localized structures attract nowadays a lot of interest since they are potential candidates for optical memories [15].

In one-dimensional systems, localized structures or localized patterns can be described as homoclinic orbits passing close to a spatially oscillatory state $[1,16]$, or to the ghost of a spatial pattern $[17,18]$, and converging to an homogeneous state, whereas domains are seen as heteroclinic trajectories joining the fixed points of the corresponding dynamical system [19]. Recently, in a nematic liquid crystal light valve with optical feedback it has been found experimentally a different type of localized states, appearing as large amplitude peaks nucleating over a lower amplitude pattern, therefore called localized peaks [20]. Figure 1 shows the typical localized peak observed in the light valve with optical feedback. More recently, similar observations have been numerically reported in other optical systems, such as in atomic vapors with optical feedback [21] and in intracavity photonic crystals [22]. Moreover, localized peaks appear also in a Newtonian fluid, when nonlinear surface waves are parametrically excited with two frequencies [23], and in monoatomic layer deposition [24]. Thus, these examples of localized states appearing over a patterned background [2024] seem to constitute a different universal class of structures with respect to the localized states that rise up from an uniform background [1, 4-9, 11-13, 16, 19].

The interplay of localized states with a structured background is particularly interesting in nonlinear optics, 


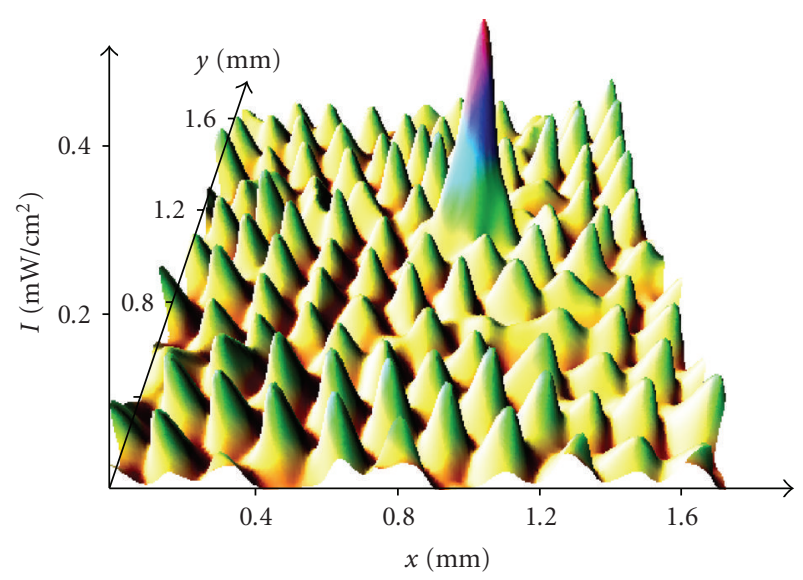

Figure 1: A typical experimental profile of a localized peak observed in the LCLV with optical feedback.

where it opens interesting technical issues on the control of localized structures, with the possibility of driving their dynamics and tailoring their interactions [25-28]. Here, we present a general description of bi-pattern systems, by which we mean systems that exhibit coexistence between two distinct pattern states and are, therefore, able to generate localized peaks. In these systems, localized structures are pinned over an underlying spatial grid, which can be either spontaneously created by a self-organized pattern formation or externally induced by a spatial periodic forcing. We show that in both cases the observed dynamical behaviors are qualitatively the same. However, while the features of the spontaneous pattern formation are difficult to control, the external forcing has the advantage of providing an easy and precise way to determine the features of localized structures, as well as to allow a direct comparison with the theoretical developments.

On the basis of amplitude equations, a first preliminary one-dimensional description of localized states observed in bi-pattern systems was done by the present authors in a recent Letter [29]. The aim of the present article is to study and characterize the universal mechanism that is at the origin of localized states pinned over an underlying lattice. In order to derive an unifying and simple description of localized peaks, we develop a theoretical model for onedimensional spatially extended systems close to a spatial bifurcation. The model, which describes coexistence of two different patterns and stable front solutions between them, is based on an amplitude equation that includes a spatial parametric forcing. This extension with respect to conventional amplitude equations, allows to describe localized patterns and to account for the main properties of these solutions. The model includes the interaction of the slowly varying envelope with the small scale of the underlying pattern [30], well-known as the nonadiabatic effect $[31,32]$.

As examples of bi-pattern systems in two-dimensions, we consider: a liquid crystal light valve (LCLV) with optical feedback, which provides our experimental framework, and a spatially forced subcritical pitchfork as a prototype model. Both systems show robust existence of localized states, which exhibit different shapes depending on the symmetry of the supporting patterns.

The paper is organized as follows. In Section 2 we present the two example systems, namely the experiment of the LCLV with optical feedback, and the forced subcritical pitchfork model, showing the typical localized states appearing in both systems. Section 3 contains the unified one-dimensional description of bi-pattern systems, which relies on the derivation of an amplitude equation amended by the inclusion of nonadiabatic terms, and Section 4 are the conclusions.

\section{Examples of Localized States in Bi-Pattern Systems}

To provide inspiration of localized states in bi-pattern systems, we shall consider a liquid crystal valve with optical feedback as an experiment and numerical simulations of a spatially forced subcritical pitchfork as a prototype model. In the experiment, the underlying lattice of the bi-pattern is realized either by letting the systems to spontaneously generate a self-organized pattern, or by imposing an intensity grid through a spatial periodic forcing. This last case compares directly with the model, which is indeed a spatially forced amplitude equation.

2.1. Experimental Evidence of Localized Peaks. The experimental setup, consisting of a LCLV in an optical feedback loop, is the same as the one reported in [33]. The LCLV is composed of a nematic liquid crystal film inserted in between a glass and a photoconductive plate over which a dielectric mirror is deposed. The liquid crystal film is planar aligned (nematic director $\vec{n}$ parallel to the walls), with a thickness $d=15 \mu \mathrm{m}$. Transparent electrodes deposited over the glass plates permit the application of an external voltage $V_{0}$ across the liquid crystal layer. The photoconductor behaves like a variable resistance, which decreases for increasing illumination. The feedback is obtained by sending back onto the photoconductor the light which has passed through the liquid-crystal layer and has been reflected by the dielectric mirror. The light beam experiences a phase shift which depends on the liquid crystal reorientation and, on its turn, modulates the effective voltage that locally applies to the liquid crystals.

The feedback loop is closed by an optical fiber bundle and is designed in such a way that diffraction and polarization interference are simultaneously present [33]. The presence of diffraction leads to the spontaneous generation of selforganized patterns, which display a typical spatial period scaling as $\sim \sqrt{\lambda L}$, where $\lambda$ is the laser wavelength and $L$ is the optical free propagation length in the feedback loop [34]. On the other hand, the presence of polarization interference leads to bistability between different spatial states. Setting $L=0$ eliminates diffraction effects, so that in this case the system exhibits bistability between homogeneous states.

As a first set of experiment, we fix $L=-40 \mathrm{~mm}$, so that the system spontaneously selects the lattices contributing to the bi-pattern interplay. The voltage applied to the LCLV has a rms value of $V_{0}=12.3 \mathrm{~V}$, with a frequency $6 \mathrm{kHz}$. Note that the period of the sinusoidal voltage $V_{0}$ is much shorter than 


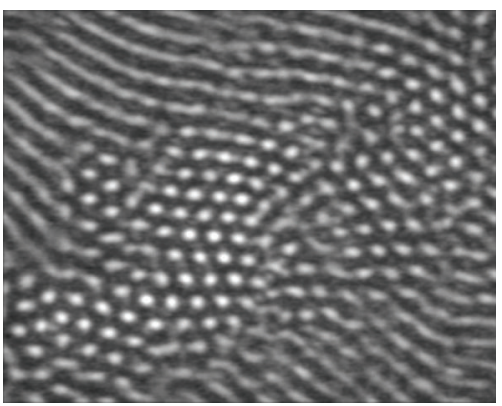

(a)

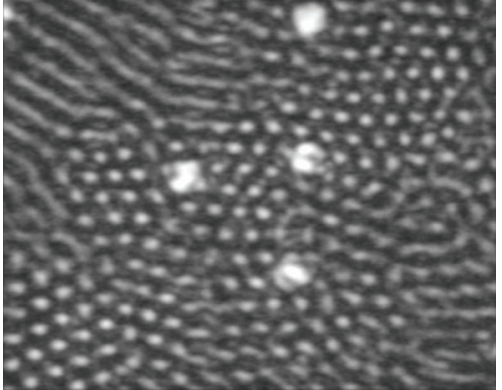

(b)

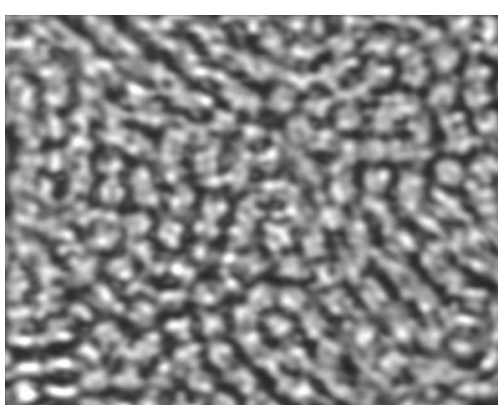

(c)

FIGURE 2: Hexagonal patterns and localized peaks observed in the LCLV system for $I_{\text {in }}=$ (a) 0.32 , (b) 0.38 and (c) $0.52 \mathrm{~mW} / \mathrm{cm}^{2}$. From $[20]$.

the liquid crystal response time and of the typical times for electroconvection [35], thus, liquid crystals are sensitive only to the rms value of the applied voltage and perform a static reorientation. Hydrodynamical effects, such as backflow, are avoided and the molecular realignment is a pure Fréedericksz transition [36].

By increasing the input light intensity $I_{\text {in }}$ we observe a sequence of transitions, as shown by the experimental snapshots of Figure 2. First, the homogeneous steady-state looses stability and develops a pattern of hexagons (Figure 2(a)). By further increasing $I_{\text {in }}$, localized peaks of higher amplitude appear over the hexagonal background (cf. Figures 1 and 2(b)). For higher values of $I_{\text {in }}$ the system exhibits a novel pattern state, which has a small coexistence region with the hexagonal pattern [20]. Figure 2(c) shows the pattern state observed for high $I_{\text {in }}$.

The theoretical model for the LCLV feedback system was previously derived in [37] and consists in two coupled equations, one for the average director tilt $\theta(\vec{r}, t), 0 \leq \theta \leq$ $\pi / 2$, and one for the feedback light intensity $I_{w}$. The equation for the director reads as

$$
\tau \partial_{t} \theta=l^{2} \nabla_{\perp}^{2} \theta-\theta+f(\theta)
$$

where $l$ is the electric coherence length, $\tau$ the local relaxation time and $f(\theta)$ a function taking into account the response of the photoconductor to the feedback intensity $I_{w}: f(\theta)=0$ when $V \leq \Gamma V_{\mathrm{FT}}$ and $f(\theta)=\pi / 2\left(1-\sqrt{\Gamma V_{\mathrm{FT}} / V}\right)$ when $V>$ $\Gamma V_{\mathrm{FT}}$, with $V$ the voltage that effectively applies to the liquid crystals

$$
V=\Gamma V_{0}+\alpha I_{w}(\theta)
$$

and $V_{\mathrm{FT}}$ the threshold voltage for the Fréedericksz transition. $\Gamma$ is the impedance of the LCLV dielectric layers and $\alpha$ a phenomenological parameter summarizing, in the linear approximation, the response of the photoconductor. After a free propagation length $L$, the feedback light intensity is given by

$$
I_{w}=\frac{I_{\text {in }}}{4}\left|e^{i(L \lambda / 4 \pi) \nabla_{\perp}^{2}}\left(1-e^{-i \beta \cos ^{2} \theta}\right)\right|^{2}
$$

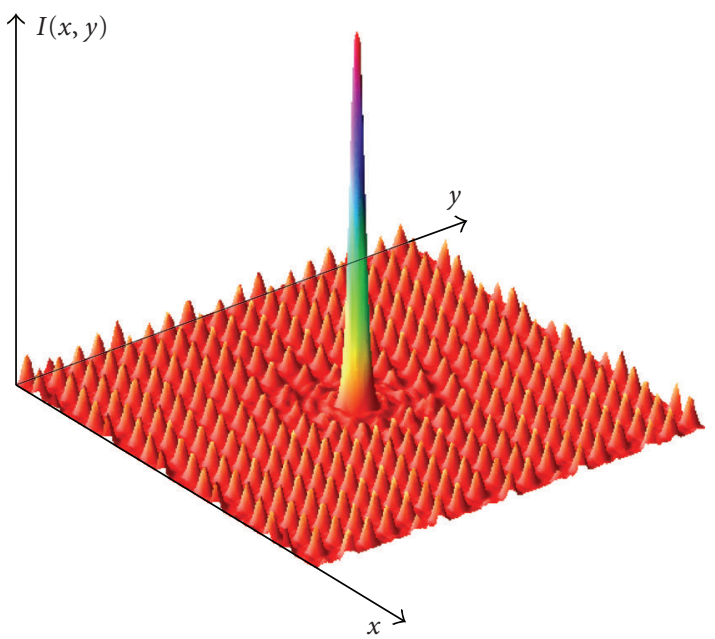

FIgURE 3: Numerical profile of the intensity showing a localized peak in the LCLV system.

the diffraction being accounted for by the operator $e^{i(L \lambda / 4 \pi) \nabla_{\perp}^{2}}$. Similar relationships between the tilt angle and the optical intensity distribution have been previously derived for light diffraction in electroconvective liquid crystal cells, where far-field diffraction [38] or shadowgraph methods [39] were employed for pattern visualization, but without any feedback of light onto the tilt angle. Recently, electro-hydrodynamic convection in a nematic liquid crystal cell with a photoconductive electrode has been reported [40]. In such a case, though there was no feedback, the light beam was acting as an external photocontrol, locally modifying the voltage applied to the liquid crystal.

We have performed numerical simulations of our model equations (1), (2), and (3) by taking as values of the parameters $\Gamma=0.15, \alpha=5.5 \mathrm{Vcm}^{2} / \mathrm{mW}, V_{\mathrm{FT}}=3.0 \mathrm{~V}$, $l=30 \mu \mathrm{m}, \lambda=632 \mathrm{~nm}, L=-40 \mathrm{~mm}$. In Figure 3 is displayed a numerical intensity profile showing a localized peak over a hexagonal pattern. By comparing with Figures 1 and 2, we can see that the numerical profile is in a fairly good agreement with the experimental profile and snapshot for the light intensity. 
In brief, it exists a large range of parameters within which the LCLV with optical feedback spontaneously is a bipattern system, exhibiting localized states pinned over a selforganized lattice generated by the system itself.

2.2. Spatially Forced Subcritical Pitchfork Model. A prototype model of bistability is the subcritical pitchfork model

$$
\partial_{t} u=\mu u+v u^{3}-u^{5}+\nabla_{\perp}^{2} u
$$

where $u(x, t)$ is a scalar field, $\mu$ is the bifurcation parameter, $v$ characterizes the type of bifurcation, which is subcritical (supercritical) for positive (negative) $v$ and $\nabla_{\perp}^{2} \equiv \partial_{x x}+\partial_{y y}$. The steady states of the above model are $u_{0}=0$ and

$$
u_{ \pm, \pm}= \pm \sqrt{\nu \pm \sqrt{v^{2}+4 \mu}}
$$

where $u=\left\{0, u_{ \pm,+}\right\}$and $u=\left\{u_{ \pm,-}\right\}$are stable and unstable uniform states, respectively. Figure 4 depicts the bifurcation diagram of subcritical pitchfork model (4) and the respective critical points that characterize the bifurcation, namely the beginning of the bistability $B$, the Maxwell point $\mu_{M}$ and the transition point $T$.

In order to have a bi-pattern system, we consider the following spatially forced model

$$
\begin{aligned}
\partial_{t} u= & \mu u+v u^{3}-u^{5}+\nabla^{2} u+a \cos (k x) \\
& +b \cos \left(k \frac{x-\sqrt{3} y}{2}\right)+c \cos \left(k \frac{x+\sqrt{3} y}{2}\right),
\end{aligned}
$$

where $\{a, b, c\}$ and $k$ are the amplitude and wave number of the spatial forcing. For small identical forcing amplitudes $(a=b=c)$ or antisymmetrical one $(b=c=-a)$, the uniform stable state of the subcritical pitchfork model (4) becomes a hexagonal or, respectively, honeycomb pattern with amplitude proportional to $a$. Hence, in the bistability region, the above spatially forced equation is a bi-pattern system with hexagonal symmetry. In Figure 5 are shown the typical hexagonal patterns observed and the interface between them.

Hence, in the bi-pattern region we observe front solutions and localized states between the two patterns. As a consequence of the interplay between the envelope variations and the wave number of the underlying pattern, the front solutions are motionless in a region of parameters, so-called the pinning range. Close to this region we expect to observe a family of localized states [30, 31]. Figure 6 illustrates a typical localized state observed in the hexagonally forced model (6), that appears as a localized peak over a structured background. In the experiment of the LCLV with feedback, it has been recently shown that the effect of a hexagonal spatial forcing induces regular arrays of localized structures [26].

To illustrate that the localized peaks exhibit different shapes depending on the supporting pattern, we consider the subcritical pitchfork model with an orthogonal forcing

$$
\partial_{t} u=\mu u+v u^{3}-u^{5}+\nabla^{2} u+a \cos (k x)+a \sin (k y),
$$

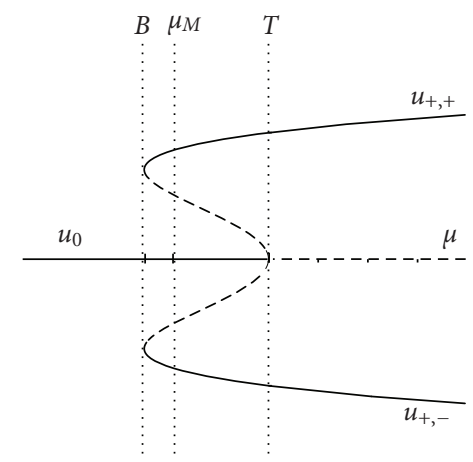

Figure 4: Bifurcation diagram of model (4), the continuous and dashed curves stand for the stable, respectively, unstable uniform state. $B, \mu_{M}$ and $T$ stand for beginning of bistability, Maxwell and transition points.

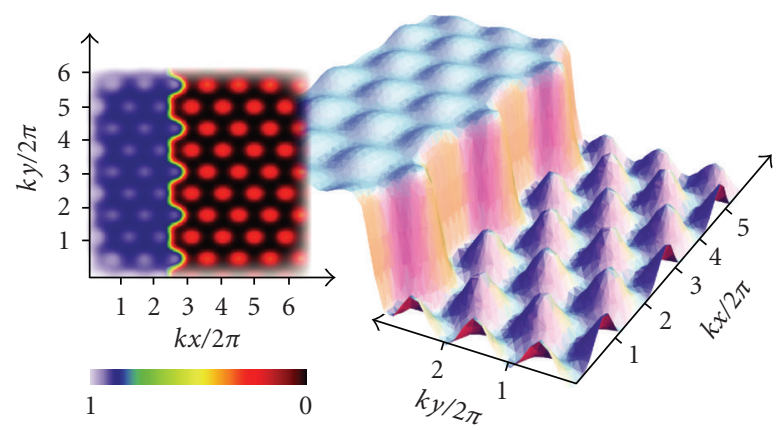

FIGURE 5: Interface between the two hexagonal patterns exhibited by model (6) with $\mu=-0.18, v=1.0$, and $a=b=c=0.02$. The inset figure shows the density plot of the field $u(x, t)$.

where $a$ is the amplitude of the spatial forcing. For small forcing, the uniform stable states of the subcritical pitchfork model (4) become square patterns with amplitude proportional to $a$. Hence, in the bistability region, the above spatially forced equation is also a bi-pattern system. In Figure 7 is shown the typical localized states observed in model (7).

\subsection{Spatially Forced Experiment: Bi-Patterns and Localized} States. Recently, we have shown in the LCLV experiment that a spatially periodic grid can be imposed, by means of a spatial light modulator, on the profile of the input beam [26]. In such a case, localized structures are pinned over the grid, which controls their dynamical behavior. Here, we have set to zero the free propagation length in the feedback loop, that is, $L=0$, hence no spatial scale is spontaneously selected by the system itself. Then, by using the previous technique, we have imposed on the input beam a spatial grid of the desired symmetry and period. In doing so, we are strongly motivated by the possibility of establishing a direct comparison with the above model, (6) or (8).

We have imposed on the system either a hexagonal or a square intensity grid. For both grids, the input intensity is $1.1 \mathrm{~mW} / \mathrm{cm}^{2}$ with an amplitude modulation of approximately $10 \%$. For the square grid, the spatial period is set to $130 \mu \mathrm{m}$ whereas for the hexagonal grid it is $150 \mu \mathrm{m}$. The 


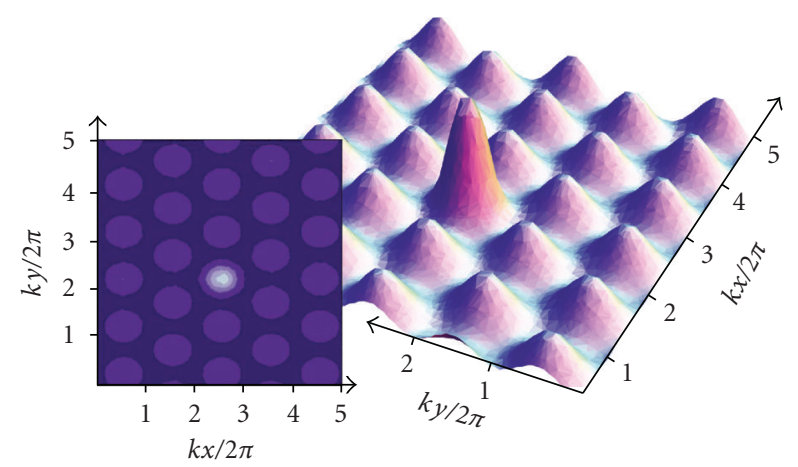

FIGURE 6: Localized peak observed in the spatially forced model (6) for a hexagonal forcing, $a=b=c=0.02$, and $\mu=-0.18, v=1.0$. The inset figure shows the corresponding density plot of the field $u(x, t)$.

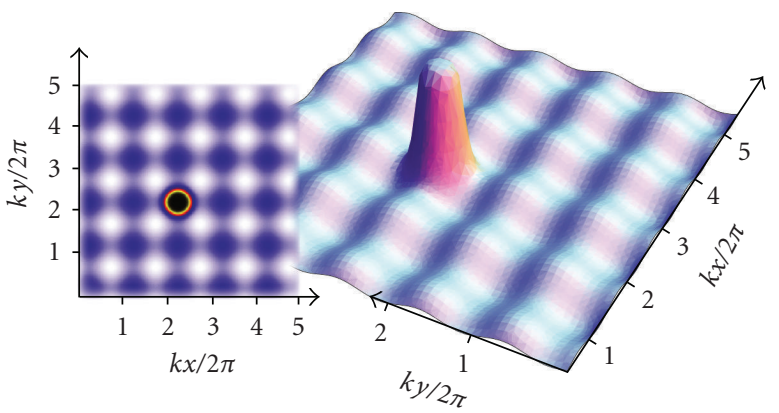

FIGURE 7: Localized peak observed in the spatially forced model (7) for an orthogonal forcing, $a=0.01$, and $\mu=-0.18, v=1.0$. The inset figure shows the corresponding density plot of the field $u(x, t)$.

voltage applied to the LCLV is slightly changed around $V_{0}=$ $5.5 \mathrm{~V}$, with a fixed frequency of $5 \mathrm{kHz}$. For this values of parameters the system is bistable and, being spatially forced by the intensity grid, it displays bi-patterns an localized states. By setting a specific initial condition, we can select either to induce an interface between the two patterns or to create localized peaks.

As examples of bi-patterns, we show in Figures 8(a) and $8(\mathrm{~b})$ the interface between a high and a low amplitude pattern obtained with (a) a square and (b) a hexagonal grid, respectively. For the same grids, but changing the initial conditions, we can easily induce localized peaks. An example of localized peak is shown in Figure 9 for a square grid.

We can notice a good qualitative agreement between the experimental profiles and those obtained by numerical simulation of the spatially forced models (6) and (7), for a hexagonal and a square forcing, respectively. Moreover, the qualitative behavior of the spatially forced systems, both the experiment and the model, is very similar to the one displayed, in a certain range of parameters, by the unforced system with diffraction playing the role of forcing. Indeed, in the region of parameters where the unforced system displays localized peaks, the qualitative dynamical features are very closely the same as those displayed by the forced systems. However, when the input intensity is increased, the unforced

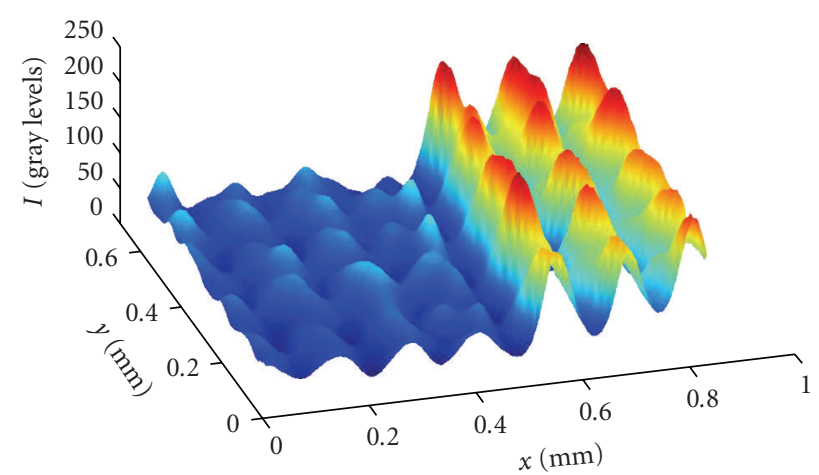

(a)

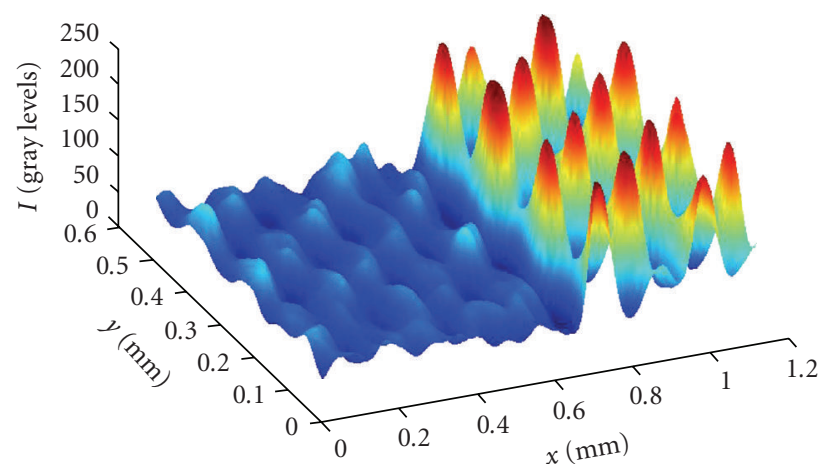

(b)

FIGURE 8: Interface between the two patterns observed in the spatially forced experiment: (a) square grid, (b) hexagonal grid. The voltage applied to the LCLV is (a) $V_{0}=5.45 \mathrm{~V}$, (b) $V_{0}=5.65 \mathrm{~V}$. The gray levels of the intensity go from zero to $1.1 \mathrm{~mW} / \mathrm{cm}^{2}$.

system shows a transition to a spatiotemporal chaotic state, which is not the case for the spatially forced system.

In conclusion, localized states in bi-pattern systems are robust phenomena and appear naturally when bistability is accompanied by a mechanism of spatial periodic forcing. This can be either externally imposed, or generated by the system itself in a certain range of parameters. In the next section we will present an unified description of localized states in one-dimensional bi-pattern systems.

\section{Unified Description}

As we have seen, the main ingredient for the appearance of localized peaks is the coexistence of two spatially periodic states, and this, in some sense, regardless the way in which the two patterns have been created. In order to provide a generic description of such a situation, we consider a a one-dimensional spatially extended system that exhibits a sequence of spatial bifurcations as shown in Figure 10, that is, the primary bifurcation is supercritical while the secondary one is of subcritical type. Let $\vec{u}(x ; t)$ be a vector field that describes the system under study and satisfies the partial differential equation

$$
\partial_{t} \vec{u}=\vec{f}\left(\vec{u}, \partial_{x},\left\{\lambda_{i}\right\}\right),
$$




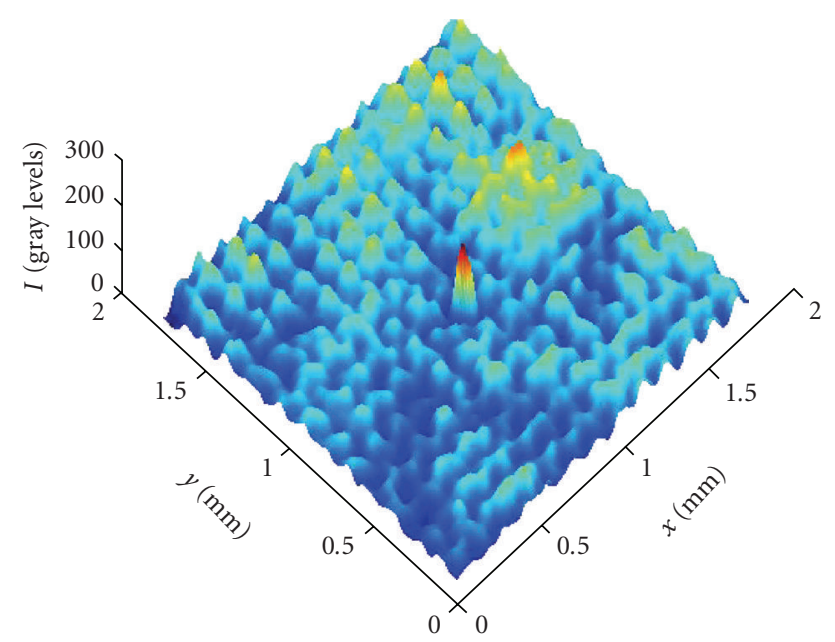

FIGURE 9: Localized peak observed in the spatially forced experiment for a square grid. The voltage applied to the LCLV is $V_{0}=$ $5.40 \mathrm{~V}$.

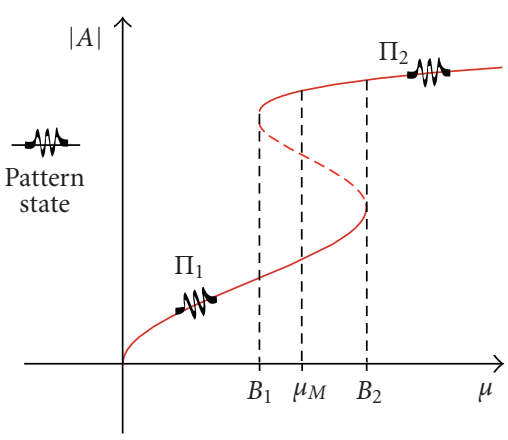

FIgURE 10: A typical bifurcation diagram allowing for the appearance of localized peaks: at a certain value of $\mu$ a secondary subcritical bifurcation takes place; dashed lines mark the beginning (end) $B_{1}$ $\left(B_{2}\right)$ of the bistable region and the Maxwell point $\mu_{M}$. From [29].

where $\left\{\lambda_{i}\right\}$ is a set of parameters. For a critical value of one of the parameters, the system exhibits a spatial instability at a given wave number $q_{c}$. Close to this spatial instability, we use the Ansatz

$$
\vec{u}=A(X, T) e^{i q_{c} x} \hat{u}+\bar{A}(X, T) e^{-i q_{c} x} \hat{\bar{u}}+\cdots
$$

and the standard amplitude equations reads as [1]

$$
\partial_{T} A=\mu A-\nu|A|^{2} A+\alpha|A|^{4} A-|A|^{6} A+\partial_{X X} A,
$$

where $\mu$ is the bifurcation parameter and $\{\nu, \alpha\}$ control the type of the bifurcation (first- or second-order depending on the sign of these coefficients). Higher-order terms are ruled out by scaling analysis, since $\nu \sim \mu^{2 / 3}, \alpha \sim \mu^{1 / 3},|A| \sim \mu^{1 / 6}$, $\partial_{t} \sim \mu, \partial_{x} \sim \mu^{1 / 2}$, and $\mu \ll 1$. Note that this approach is phase invariant $\left(A \rightarrow A e^{i \varphi}\right)$, but the initial system under study does not necessarily have this symmetry.

As depicted in Figure 10, for a given range of parameter values the system shows coexistence between two different spatially periodic states, each one corresponding to a homogeneous state for the amplitude equation. Thus, it is a bi-pattern system. The coexistence region is $B_{1}<$ $\mu<B_{2}$. The extended stationary solution of the amplitude equation (10), has the form $A=R(x) e^{i \theta(x)}$, where $R(x)$ and $\theta(x)$ are the envelope modulus and phase, respectively. These functions satisfy the relation $\theta(x)=\int \epsilon / R(x)^{2} d x$. For uniform modulus solution $\left(R_{o}\right)$, one has the expression for the envelope

$$
A=R_{o} e^{i\left(\gamma / R_{o}^{2}\right) X}
$$

with

$$
\mu-\frac{\gamma^{2}}{R_{o}^{4}}-v R_{o}^{2}+\alpha R_{o}^{4}-R_{o}^{6}=0,
$$

and $\gamma$ is an arbitrary constant related to the initial phase invariance. It is worth to note that in the case of positive $\gamma$, the wave number of the pattern is modified by the inverse of the square amplitude $R_{0}^{2}$, so that patterns with larger amplitude have smaller wave number. At variance, when $\gamma$ is negative the patterns with larger amplitude have smaller wavelength [29].

For given values of the parameters, the two stable uniform stationary states of (10) have the same energy, that is, the system is at the Maxwell point, where the front between the two states is motionless [41]. By moving away from the Maxwell point, the front dynamics is usually characterized by the motion of the core of the front, which is defined as the front position with the largest slope. In order to have a localized states, we consider the interaction of two of these motionless fronts close to the Maxwell point. As a consequence of the asymptotic behavior of the front at infinity, the front interaction is attractive, and has the form [42]

$$
\dot{\Delta}=-a e^{-\lambda \Delta}+\delta
$$

where $\Delta$ is the distance between the cores of each front, $\delta$ is the separation from the Maxwell point, which is proportional to $\mu-\mu_{M}, \lambda$ characterizes the exponential decay of the front to a given constant value at infinity, and $a$ is a positive coefficient that characterizes the properties of the interaction and is determined by the form of the front. The interaction law (13) has an unstable fixed point $\Delta^{*}=-\ln (\delta / a) / \lambda$, which is the nucleation barrier between the two homogeneous states. Hence, the conventional amplitude equation, (10), does not exhibit stable localized states, due to the scale separation used to derive the amplitude equation. But near the front's core, the previous Ansatz is no more valid. Indeed, in these locations the slowly varying envelope $A(X, T)$ shows oscillations of the same (or comparable) size as the small scale of the underlying pattern. This phenomenon is denominated as the nonadiabatic effect [30-32].

3.1. Amended Amplitude Equation. In order to take into account the nonadiabatic effect, we compute the corrections of the amplitude equation by including the nonresonant 
terms, that is, the solvability condition for the amplitude $A$ has the form

$$
\begin{aligned}
\partial_{T} A= & \frac{q_{c}}{2 \pi} \int_{X}^{X+2 \pi / q_{c}} f\left(|A|^{2}, \partial_{X X}\right) A+\partial_{X X} A d x \\
& +\sum_{m-n=1}^{N} \frac{g_{m n} q_{c}}{2 \pi} \int_{X}^{X+2 \pi / q_{c}} A^{m} \bar{A}^{n} e^{-\left(i q_{c}(1+n-m) x / \sqrt{\mu}\right)} d x
\end{aligned}
$$

with $f\left(|A|^{2}\right)=\mu-\nu|A|^{2}+\alpha|A|^{4}-|A|^{6}+O\left(|A|^{8}, \bar{A} \partial_{X X} A\right)$, $O\left(|A|^{8}, \bar{A} \partial_{X X} A\right)$ stands for high order terms, $m, n \geq 0, g_{m n}$ are real numbers of order one and $N$ is the degree of highest nonlinearity. The resonant terms are obtained by imposing a solvability condition, where one assumes that there is a scale separation between the spatial variation of the envelope and that of the underlying pattern, that is, the spatial variation of the envelope is large enough with respect to the pattern wavelength $\left(\partial_{X} A \ll q_{c} A\right)$. Hence in this limit, we can approach

$$
\begin{gathered}
\frac{q_{c}}{2 \pi} \int_{X}^{X+2 \pi / q_{c}} f\left(|A|^{2}\right) A d x \approx f\left(|A|^{2}\right) A, \\
\frac{q_{c}}{2 \pi} \int_{X}^{X+2 \pi / q_{c}} A^{m} \bar{A}^{n} e^{-\left(i q_{c}(1+n-m) x / \sqrt{\mu}\right)} d x \approx 0 .
\end{gathered}
$$

However, the above assumption is often not satisfied close to the front core. To take into account this coupling, we can compute the integral of the solvability condition with the stationary phase method, thus

$$
\begin{aligned}
& \frac{q_{c}}{2 \pi} \int_{X}^{X+2 \pi / q_{c}} A^{m} \bar{A}^{n} e^{-\left(i q_{c}(1+n-m) x\right) / \sqrt{\mu}} d x \\
& \left.\quad \approx \frac{\sqrt{\mu}}{i q_{c}(m-n-1)} \frac{A^{m} \bar{A}^{n} e^{-\left(i q_{c}(1+n-m) / \sqrt{\mu}\right) x}}{2 \pi / q_{c}}\right|_{X} ^{X+2 \pi / q_{c}} \\
& \quad \approx \frac{\sqrt{\mu} \partial_{X}\left[A^{m} \bar{A}^{n}\right]}{i q_{c}(m-n-1)} e^{-\left(i q_{c}(1+n-m) / \sqrt{\mu} X\right.},
\end{aligned}
$$

which allows us to take into account the effect of nonresonant terms. Finally, the amended amplitude equation reads as [43]

$$
\begin{aligned}
\partial_{T} A= & \mu A-\nu|A|^{2} A+\alpha|A|^{4} A-|A|^{6} A+\partial_{X X} A \\
& +\sqrt{\mu} \sum_{m, n \geq 0}^{N} g_{m n} \frac{\partial_{X}\left[A^{m} \bar{A}^{n}\right]}{i q_{c}(m-n-1)} e^{-i\left(q_{c}(1+n-m) / \sqrt{\mu}\right) X},
\end{aligned}
$$

where $g_{m n}$ are real numbers of order one and $N$ is the degree of highest nonlinearity. Hence, the resulting amplitude equation is parametrically forced in space by the nonresonant terms, which are higher order terms with the asymptotic scaling under consideration. It is important to remark that the nonresonant terms do not change the uniform states, because these terms are proportional to the spatial derivative of the envelope. Notice that the Ansatz for $\vec{u}$ satisfies the symmetries $\{x \rightarrow-x, A \rightarrow \bar{A}\}$, and $\left\{x \rightarrow x+x_{o}, A \rightarrow\right.$ $\left.A e^{i q_{c} x_{0}}\right\}$, thus restoring the original symmetry, while the spatial translation and phase invariance are independent symmetries of (10). Recently, we have derived a phenomenological model in which the spatial forcing has an amplitude proportional to a polynomial development of the slowly varying amplitude [29]. In this case, the nonadiabatic effect is overestimated since the nonresonant terms are proportional to $\sqrt{\mu}$, however the qualitative dynamics of both models are very similar.

To illustrate the effect of nonresonant terms we keep the leading term $n=0$ and $m=2$. Then the forced amplitude equation takes the form

$$
\begin{aligned}
\partial_{T} A= & \mu A-\nu|A|^{2} A+\alpha|A|^{4} A-|A|^{6} A+\partial_{X X} A \\
& +\sqrt{\mu} \eta \frac{A \partial_{X} A}{i q_{c}} e^{i\left(q_{c} / \sqrt{\mu}\right) X} .
\end{aligned}
$$

The slowly varying amplitude is now spatially forced with a frequency $q_{c} / 2 \pi \sqrt{\mu}$ and an amplitude proportional to $\eta \equiv g_{02}$. As a consequence of the spatial forcing, the front solution between the spatially periodic states exhibits a pinning range, that is, the front is motionless for a range of parameters around the Maxwell point. We have to note that the amplitude of the forcing is proportional to the gradient of the slowly varying amplitude, $\partial_{X} A$, thus the forcing is effective at the interfaces, where the amplitude changes rapidly, and zero elsewhere.

In order to obtain the change of the front interaction as a result of the spatial forcing, we consider the front solution of the resonant equation

$$
A_{ \pm}\left(x-x_{o}\right)=R_{ \pm}\left(x-x_{o}\right) e^{i \int \varepsilon / R_{ \pm}^{2}, d x}
$$

where $R_{ \pm}\left(x-x_{o}\right)$ satisfies

$$
\mu R-\nu R^{3}+\alpha R^{5}-R^{7}+\partial_{x x} R-\frac{\varepsilon^{2}}{R^{3}}=0,
$$

$x_{o}$ is the position of the front core and the lower index $+(-)$ corresponds to a front monotonically rising (decreasing). As the nonresonant term is a rapid spatial oscillation, we consider this term as perturbative-type and use the Anstaz

$$
\begin{aligned}
A= & A_{+}\left(x-x_{1}(t)\right)+A_{-}\left(x-x_{2}(t)\right) \\
& -\left(A_{o,+}-A_{o,-}\right)+\delta W e^{i \delta \varphi}
\end{aligned}
$$

in (18), where $A_{o, \pm}=R_{o, \pm} e^{i \varepsilon x / R_{o, \pm}^{2}}$, and $\{\delta W, \delta \varphi\}$ are small functions, and $R_{o, \pm}$ are the stable equilibrium states of the resonant amplitude equation (10) and $R_{o,+}>R_{o,-}$. After straightforward calculations, we obtain the following solvability condition for the $\delta W$ function (front interaction law)

$$
\dot{\Delta}=-a e^{-\lambda \Delta}+\delta+\gamma \cos \left(\frac{q_{c}}{\sqrt{\mu}} \Delta\right),
$$




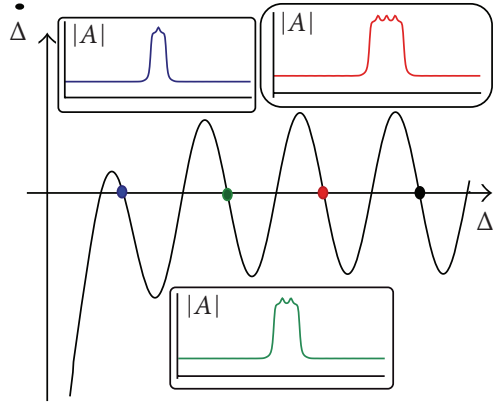

Figure 11: Oscillatory interaction force between two front solutions. The inset figures are the stable localized patterns observed at the Maxwell points (black dots), where the interaction changes its sign.

with

$$
\begin{aligned}
& a=\frac{-2\left\langle 3 \mu R_{+}^{2}-5 \nu R_{+}^{4}+7 \alpha R_{+}^{6}-3 \varepsilon R_{+}^{-4} \mid \partial_{x} R_{+}\right\rangle}{\left\langle\partial_{x} R_{+} \mid \partial_{x} R_{+}\right\rangle}, \\
& \delta=\frac{F\left(R_{+}\right)-F\left(R_{-}\right)}{\left\langle\partial_{x} R_{+} \mid \partial_{x} R_{+}\right\rangle}, \\
& \gamma \approx \frac{\sqrt{\mu} \eta\left\langle\partial_{x} R_{+}^{2} \mid R_{+} \sin \left(\left(q_{c} / \sqrt{\mu}\right) x\right)\right\rangle}{q_{c}\left\langle\partial_{x} R_{+} \mid \partial_{x} R_{+}\right\rangle},
\end{aligned}
$$

where

$$
F(R)=\frac{\mu R^{2}}{2}-\frac{\nu R^{4}}{4}+\frac{\alpha R^{6}}{6}-\frac{R^{8}}{8}+\frac{2 \varepsilon^{2}}{R^{2}},
$$

and $\langle f \mid g\rangle \equiv \int_{-\infty}^{\infty} f(x) g(x) d x$.

As a consequence of the spatial forcing the front interaction law close to the pinning range, (22), has an extra term and now alternates between attractive and repulsive forces. It is important to remark that $\sqrt{\mu} \gamma$ is a parameter exponentially small, proportional to $\eta$, and is of order $\delta$, that is, the source of the periodical force is the spatial forcing in the (18). Therefore, close to the Maxwell point the system exhibits a family of equilibrium points, $d \Delta / d t=0$. Each equilibrium point correspond to a localized solution nucleating over a pattern state. These solutions correspond to localized patterns. The lengths of localized patterns are multiple of a basic length, corresponding to the shortest localized state. These shortest states are the localized-peaks, corresponding to the experimental observations reported in [20]. In Figure 11, it is depicted the front interaction law and the family of equilibrium points.

Due to the oscillatory nature of the front interaction, which alternates between attractive and repulsive forces (cf. Figure 11), we can deduce the dynamical evolution and bifurcation diagram of localized patterns. By decreasing $\delta$ or increasing $\eta$, the family of localized patterns disappears by successive saddle-node bifurcations and only localized peaks survive.

\section{Conclusions}

Bi-pattern systems are spatially extended systems that display coexistence of two pattern states. They exhibit a rich variety of localized solutions, in particular, localized peaks are pinned over a spatial grid, that can be either spontaneously generated by the system itself, or externally imposed. These localized states are of particular interest in nonlinear optics, where they constitute the elementary pixels of optical memories. The interplay with a structured background open new technical perspectives on the control of their dynamics. We have derived an unified and simple description of localized peaks in one-dimensional spatially extended systems close to a spatial bifurcation. This model allows us to understand the mechanism underlying the formation of localized states, which is based on the coupling between the spatial variations of the envelope and the wavelength of the background pattern. In two-dimensions, we show that these localized states persist and exhibit different shapes depending on the symmetry of the supporting patterns. However, a natural extension of the front interaction law, for instance an interface tension, is not yet available. Work in this direction is in progress.

\section{Acknowledgments}

The simulation software $\operatorname{Dim} X$, developed at INLN, has been used for all the numerical simulations presented in this paper. The second author acknowledges the financial support of FONDECYT Project 1090045, and FONDAP Grant 11980002. The third author thanks the financial support FONDECYT Project 11080286. The first and the fourth authors thank the financial support of the ANR-07BLAN-0246-03, turbonde.

\section{References}

[1] M. C. Cross and P. C. Hohenberg, "Pattern formation outside of equilibrium," Reviews of Modern Physics, vol. 65, no. 3, pp. 851-1112, 1993.

[2] S. H. Strogatz, Nonlinear Dynamics and Chaos: With Applications to Physics, Biology, Chemistry and Engineering, AddisonWesley, Reading, Mass, USA, 1994.

[3] S. Wiggins, Introduction to Applied Nonlinear Dynamical Systems and Chaos, Springer, New York, NY, USA, 2003.

[4] H. A. Eschenfelder, Magnetic Bubble Technology, Springer, Berlin, Germany, 1981.

[5] S. Pirkl, P. Ribière, and P. Oswald, "Forming process and stability of bubble domains in dielectrically positive cholesteric liquid crystals," Liquid Crystals, vol. 13, no. 3, pp. 413-425, 1993.

[6] Y. A. Astrov and Y. A. Logvin, "Formation of clusters of localized states in a gas discharge system via a self-completion scenario,” Physical Review Letters, vol. 79, no. 16, pp. 29832986, 1997.

[7] K.-J. Lee, W. D. McCormick, J. E. Pearson, and H. L. Swinney, "Experimental observation of self-replicating spots in a reaction-diffusion system," Nature, vol. 369, no. 6477, pp. 215-218, 1994. 
[8] W. S. Edwards and S. Fauve, "Patterns and quasi-patterns in the Faraday experiment," Journal of Fluid Mechanics, vol. 278, pp. 123-148, 1994.

[9] P. B. Umbanhowar, F. Melo, and H. L. Swinney, "Localized excitations in a vertically vibrated granular layer," Nature, vol. 382, no. 6594, pp. 793-796, 1996.

[10] M. G. Clerc, P. Cordero, J. Dunstan, et al., "Liquid-solid-like transition in quasi-one-dimensional driven granular media," Nature Physics, vol. 4, no. 3, pp. 249-254, 2008.

[11] R. Heinrichs, G. Ahlers, and D. S. Cannell, "Traveling waves and spatial variation in the convection of a binary mixture," Physical Review A, vol. 35, no. 6, pp. 2761-2764, 1987.

[12] P. Kolodner, D. Bensimon, and C. M. Surko, "Traveling-wave convection in an annulus," Physical Review Letters, vol. 60, no. 17, pp. 1723-1726, 1988.

[13] D. W. Mc Laughlin, J. V. Moloney, and A. C. Newell, "Solitary waves as fixed points of infinite-dimensional maps in an optical bistable ring cavity," Physical Review Letters, vol. 51, no. 2, pp. 75-78, 1983.

[14] N. N. Rosanov and G. V. Khodova, "Autosolitons in bistable interferometers," Optics and Spectroscopy, vol. 65, pp. 449-450, 1988.

[15] M. Tlidi, P. Mandel, and R. Lefever, "Localized structures and localized patterns in optical bistability," Physical Review Letters, vol. 73, no. 5, pp. 640-643, 1994.

[16] P. Coullet, C. Riera, and C. Tresser, "Stable static localized structures in one dimension," Physical Review Letters, vol. 84, no. 14, pp. 3069-3072, 2000.

[17] U. Bortolozzo, M. G. Clerc, and S. Residori, submitted to New Journal of Physics.

[18] U. Bortolozzo, M. G. Clerc, and S. Residori, "Local theory of the slanted homoclinic snaking bifurcation diagram," Physical Review E, vol. 78, no. 3, Article ID 036214, 4 pages, 2008.

[19] W. van Saarloos and P. C. Hohenberg, "Pulses and fronts in the complex Ginzburg-Landau equation near a subcritical bifurcation," Physical Review Letters, vol. 64, no. 7, pp. 749$752,1990$.

[20] U. Bortolozzo, R. G. Rojas, and S. Residori, "Spontaneous nucleation of localized peaks in a multistable nonlinear system," Physical Review E, vol. 72, no. 4, Article ID 045201, 4 pages, 2005.

[21] Y. A. Logvin, B. Schäpers, and T. Ackemann, "Stationary and drifting localized structures near a multiple bifurcation point," Physical Review E, vol. 61, no. 4, pp. 4622-4625, 2000.

[22] D. Gomila and G.-L. Oppo, "Subcritical patterns and dissipative solitons due to intracavity photonic crystals," Physical Review A, vol. 76, no. 4, Article ID 043823, 7 pages, 2007.

[23] H. Arbell and J. Fineberg, "Temporally harmonic oscillons in Newtonian fluids," Physical Review Letters, vol. 85, no. 4, pp. 756-759, 2000.

[24] M. G. Clerc, E. Tirapegui, and M. Trejo, "Pattern formation and localized structures in monoatomic layer deposition," The European Physical Journal, vol. 146, no. 1, pp. 407-425, 2007.

[25] P. L. Ramazza, E. Benkler, U. Bortolozzo, S. Boccaletti, S. Ducci, and F. T. Arecchi, "Tailoring the profile and interactions of optical localized structures," Physical Review E, vol. 65, no. 6, Article ID 066204, 4 pages, 2002.

[26] U. Bortolozzo and S. Residori, "Storage of localized structure matrices in nematic liquid crystals," Physical Review Letters, vol. 96, no. 3, Article ID 037801, 4 pages, 2006.
[27] F. Pedaci, S. Barland, E. Caboche, et al., "All-optical delay line using semiconductor cavity solitons," Applied Physics Letters, vol. 92, no. 1, Article ID 011101, 3 pages, 2008.

[28] C. Cleff, B. Gütlich, and C. Denz, "Gradient induced motion control of drifting solitary structures in a nonlinear optical single feedback experiment," Physical Review Letters, vol. 100, no. 23, Article ID 233902, 4 pages, 2008.

[29] U. Bortolozzo, M. G. Clerc, C. Falcon, S. Residori, and R. G. Rojas, "Localized states in bistable pattern-forming systems," Physical Review Letters, vol. 96, no. 21, Article ID 214501, 4 pages, 2006.

[30] M. G. Clerc and C. Falcon, "Localized patterns and hole solutions in one-dimensional extended systems," Physica A, vol. 356, no. 1, pp. 48-53, 2005.

[31] D. Bensimon, B. I. Shraiman, and V. Croquette, "Nonadiabatic effects in convection," Physical Review A, vol. 38, no. 10, pp. 5461-5464, 1988.

[32] Y. Pomeau, "Front motion, metastability and subcritical bifurcations in hydrodynamics," Physica D, vol. 23, no. 1-3, pp. 3-11, 1986.

[33] S. Residori, "Patterns, fronts and structures in a liquid-crystallight-valve with optical feedback," Physics Reports, vol. 416, no. 5-6, pp. 201-272, 2005.

[34] E. Pampaloni, S. Residori, and F. T. Arecchi, "Roll-hexagon transition in a Kerr-like experiment," Europhysics Letters, vol. 24, no. 8, pp. 647-652, 1993.

[35] L. Kramer and W. Pesch, "Electrohydrodynamic instabilities in nematic liquid crystals in pattern formation in liquid crystals," in Pattern Formation in Liquid Crystals, A. Buka and L. Kramer, Eds., pp. 221-255, Springer, New York, NY, USA, 1996.

[36] P. G. de Gennes and J. Prost, The Physics of Liquid Crystals, Oxford Science, Clarendon Press, Oxford, UK, 2nd edition, 1993.

[37] M. G. Clerc, A. Petrossian, and S. Residori, "Bouncing localized structures in a liquid-crystal light-valve experiment," Physical Review E, vol. 71, no. 1, Article ID 015205, 4 pages, 2005.

[38] T. O. Carroll, "Liquid-crystal diffraction grating," Journal of Applied Physics, vol. 43, no. 3, pp. 767-770, 1972.

[39] S. Rasenat, G. Hartung, B. L. Winkler, and I. Rehberg, "The shadowgraph method in convection experiments," Experiments in Fluids, vol. 7, no. 6, pp. 412-420, 1989.

[40] M. Henriot, J. Burguete, and R. Ribotta, "Entrainment of a spatially extended nonlinear structure under selective forcing," Physical Review Letters, vol. 91, no. 10, Article ID 104501, 4 pages, 2003.

[41] P. Collet and J. P. Eckmann, Instabilities and Fronts in Extended Systems, Princeton University Press, Princeton, NJ, USA, 1990.

[42] K. Kawasaki and T. Ohta, "Kink dynamics in one-dimensional nonlinear systems," Physica A, vol. 116, no. 3, pp. 573-593, 1982.

[43] M. G. Clerc, S. Coulibaly, D. Escaff, C. Falcón, C. Fernandez, and R. G. Rojas, in preparation. 

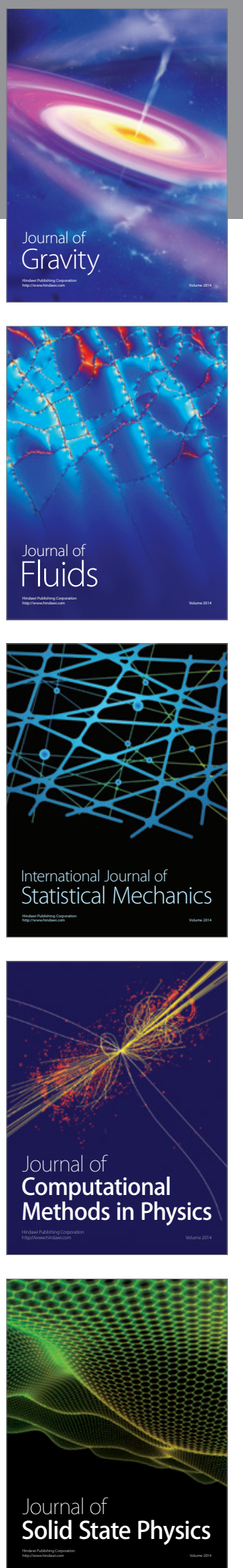

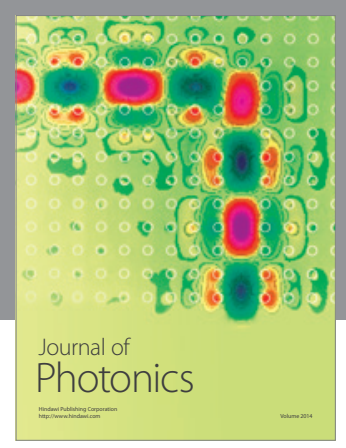

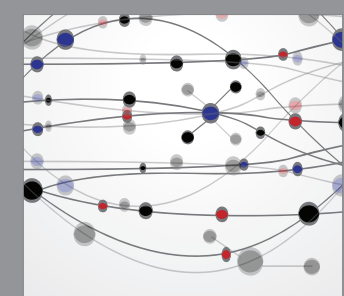

The Scientific World Journal
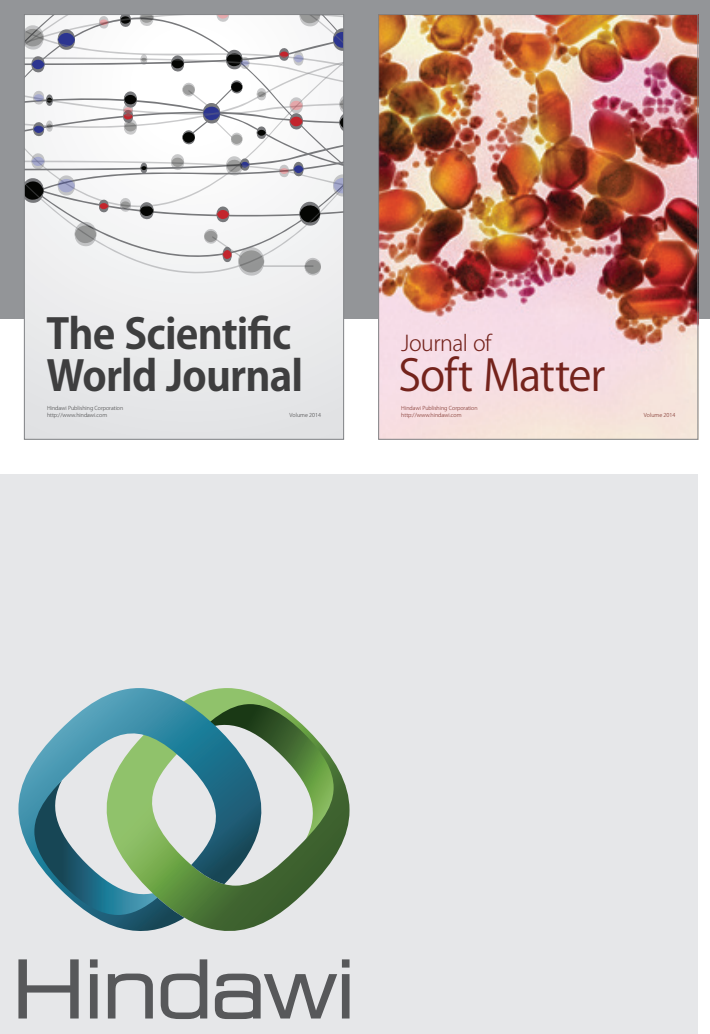

Submit your manuscripts at

http://www.hindawi.com
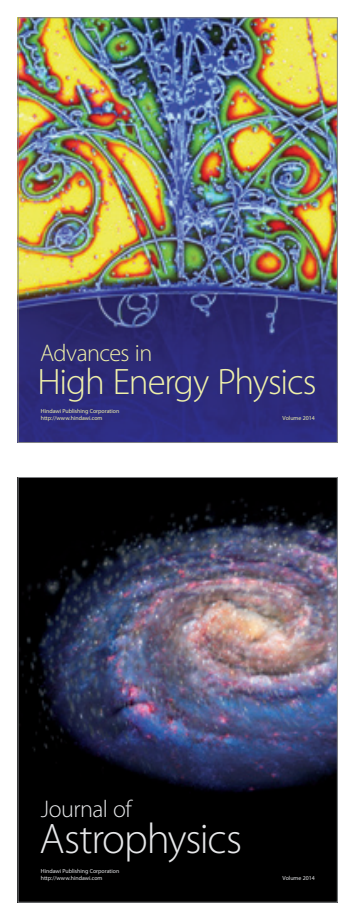
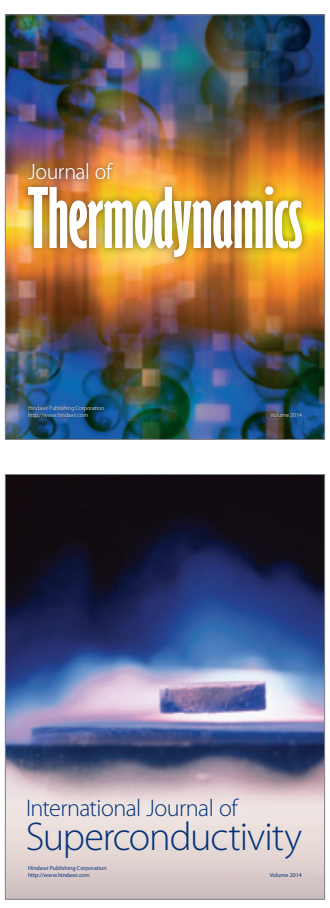
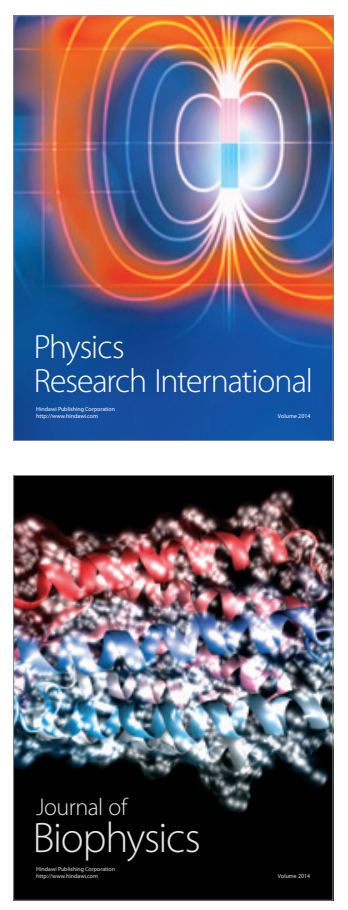
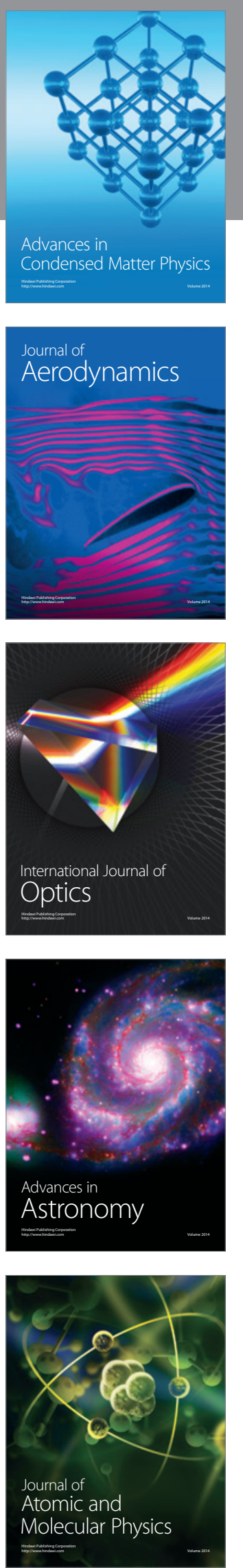